\title{
Kinetics of Fatty Acid Biohydrogenation In Vitro'
}

\author{
C. V. D. M. Ribeiro, M. L. Eastridge, ${ }^{2}$ J. L. Firkins, N. R. St-Pierre, and D. L. Palmquist \\ Department of Animal Sciences, The Ohio State University, Columbus 43210
}

\begin{abstract}
Biohydrogenation (BH) of fatty acids (FA) from fresh alfalfa and alfalfa hay with varying levels of supplemental sucrose and media $\mathrm{pH}$ was evaluated in vitro. A multicompartmental model was then developed to estimate pool size and flux of vaccenic acid (VA) during $\mathrm{BH}$ of FA in fresh alfalfa. To vary incubation $\mathrm{pH}$, alfalfa samples were inoculated with rumen fluid in 2 media differing in molarity of the bicarbonate buffer. Samples were incubated for $0,1,2,3,4,6,9$, and $12 \mathrm{~h}$; $\mathrm{pH}$ was measured and tubes were put in ice and stored until analysis. The BH rates of linoleic acid (18:2) and linolenic acid (18:3) were estimated by PROC NLIN of SAS (single pool, first-order kinetic model) and SAAM II (multiple pools, first-order kinetic model). Both methods gave similar estimates for the $\mathrm{BH}$ rates of $18: 2$ and 18:3 as well as the temporal pool size of VA. The BH rates $(\% / \mathrm{h})$ in the strong (SB) and weak buffers (WB) were $27.4( \pm 0.7)$ and $23.5( \pm 0.9)$ for $18: 2$, and $43.8( \pm 0.2)$ and $30.3( \pm 0.6)$ for $18: 3$, respectively. The WB decreased the $\mathrm{BH}$ rates of $18: 2$ and 18:3 for both forage sources. However, $\mathrm{BH}$ rates of 18:3 were higher from fresh alfalfa than alfalfa hay. There was no effect of sucrose addition on the $\mathrm{BH}$ rates of $18: 2$ and 18:3. Moreover, there was no effect of buffer on the BH of VA estimated by the multiple pools model between the SB and WB $(12.5 \pm 2.1$ and $14.1 \pm 3.7 \% / \mathrm{h}$, respectively). The $\mathrm{BH}$ rates of the conjugated linoleic acid isomers were not different between the SB and WB treatments (36.7 \pm 19.8 and $25.9 \pm 27.2$, respectively). Because we could estimate fluxes as well as mass of the VA pools, more information was generated from the data when a multiple pools model was used compared with a single pool, first-order kinetic model.
\end{abstract}

Key words: alfalfa, kinetics of biohydrogenation, fatty acid, multicompartmental model

\footnotetext{
Received July 22, 2006.

Accepted October 27, 2006.

${ }^{1}$ Research was supported by State and Federal funds appropriated to the Ohio Agricultural Research and Development Center, The Ohio State University.

${ }^{2}$ Corresponding author: eastridge.1@osu.edu
}

\section{INTRODUCTION}

The ruminal biohydrogenation (BH) process is responsible for alteration of dietary fatty acids (FA) in the rumen, decreasing the proportion of unsaturated FA (UFA) and the appearance of numerous unsaturated isomers, including conjugated linoleic acid (CLA) and trans 18:1 FA. Although the absorbed FA isomers affect animal performance and milk quality, quantitative flow of individual isomers is not predictable. Estimating ruminal $\mathrm{BH}$ rates can be a useful tool to quantify relationships between dietary or ruminal factors and the profile of FA reaching the duodenum of ruminants.

Grazing cows produce milk with higher concentration of CLA than those fed preserved forages (Jahreis et al., 1997; Kelly et al., 1998; French et al., 2000). Kay et al. (2004) reported that the majority of CLA in milk from cows fed pasture was derived from desaturation of vaccenic acid (trans-11 18:1; VA) by stearoyl-CoA desaturase (EC 1.14.19.1) in the mammary gland. Whereas desaturation of VA in the mammary gland contributes the majority of CLA in milk fat, less is understood concerning factors that regulate the amount of VA produced in the rumen. The higher concentration of CLA in milk fat from grazing cows has been proposed to be a result of higher levels of soluble sugars in fresh plants compared with preserved forages (Kelly et al., 1998; French et al., 2000). Concentration of sugars in grasses average $17 \%$ of DM; fructosans occur in major amounts, followed by sucrose, fructose, and glucose (Woolford, 1984). However, ensiling and drying decrease the amount of FA (Chilliard et al., 2000) and sugars in forages (Van Soest, 1994).

Kolver and de Veth (2002) reported ruminal $\mathrm{pH}$ to be lower than 6.2 for cows fed pasture-based diets. A lower $\mathrm{pH}$ may inhibit the final step of $\mathrm{BH}$, favoring formation of monoenoic trans 18:1 FA (Qiu et al., 2004). Therefore, interactions among $\mathrm{pH}$ and concentrations of 18:3 and sucrose may play a role in the overall $\mathrm{BH}$ process (Ribeiro et al., 2005).

We are not aware of any published data reporting the $\mathrm{BH}$ rates of individual trans 18:1 FA and CLA isomers. Understanding how ruminal $\mathrm{BH}$ is regulated, even in in vitro conditions, may further advance our ability to develop methods for estimating the profile of FA reaching the duodenum of ruminants. A multicompart- 
mental model approach may allow us to characterize the influence of dietary and ruminal factors on the $\mathrm{BH}$ rates of FA. Because all pathways of ruminal $\mathrm{BH}$ have not yet been defined, such an approach may also allow for improvement in understanding the $\mathrm{BH}$ pathways proposed in the literature by identifying segments of the model for which more data are needed, and therefore, stimulate further research in this area.

We postulated that the $\mathrm{BH}$ rate of UFA in fresh alfalfa is greater than that in alfalfa hay and that decreasing $\mathrm{pH}$ will decrease the $\mathrm{BH}$ rate independent of the forage preservation method. We also postulated that an interaction between $\mathrm{pH}$ and sucrose concentration also influences $\mathrm{BH}$ of 18:3 from alfalfa hay. To quantify the effect of $\mathrm{pH}$ on $\mathrm{BH}$ rates, we generated a multicompartmental model to estimate the $\mathrm{BH}$ rates, FA pool size over time, and flux of all FA during in vitro incubation of forages.

\section{MATERIALS AND METHODS}

\section{Treatments and Incubation Procedures}

Fresh alfalfa samples (prebloom/early bloom) were obtained from a greenhouse at the Ohio Agricultural Research and Development Center (Wooster, OH). Samples were harvested randomly and immersed immediately in liquid nitrogen. Samples were freezedried, ground to pass a $1-\mathrm{mm}$ screen, and stored at $-20^{\circ} \mathrm{C}$. Purchased alfalfa hay was ground to $1 \mathrm{~mm}$ and stored at $-20^{\circ} \mathrm{C}$.

Treatments were similar to those from a previous experiment in which the effect of sucrose was also tested using continuous culture fermenters (Ribeiro et al., 2005); the treatments were 1) fresh alfalfa, 2) alfalfa hay, 3) alfalfa hay plus $4 \%$ sucrose, and 4) alfalfa hay plus $8 \%$ sucrose. The concentrations of nutrients from each forage source are shown in Table 1.

The $\mathrm{pH}$ of incubations was varied by using different molarities of buffer [strong buffer (SB), $0.4 \mathrm{~mol} / \mathrm{L}$ $\mathrm{NaHCO}_{3}$; weak buffer (WB), $0.2 \mathrm{~mol} / \mathrm{L} \mathrm{NaHCO}_{3}$ ]; sodium molarities of the buffers were equalized by adding $\mathrm{NaCl}$ to the weak buffer (Piwonka and Firkins, 1996). Each substrate ( $0.5 \mathrm{~g}$ of dried forage) was incubated with SB or WB in 2 sets (blocks) of incubations.

Ruminal fluid was obtained from 2 ruminally cannulated dairy cows per set of incubation, one consuming an alfalfa hay diet and the other consuming a TMR diet consisting of $60 \%$ (wt/wt) forage (alfalfa hay plus corn silage). These were mixed to increase diversity of microorganisms present during incubation. Ruminal contents were strained through 2 layers of cheesecloth to retain small particles, which are the reservoir for fibrolytic bacteria that contribute to $\mathrm{BH}$. The ruminal fluids from 2 cows were mixed together and taken to the labo-
Table 1. Nutrient composition of fresh alfalfa and alfalfa hay

\begin{tabular}{|c|c|c|}
\hline Item & $\begin{array}{l}\text { Fresh } \\
\text { alfalfa }\end{array}$ & $\begin{array}{c}\text { Alfalfa } \\
\text { hay }\end{array}$ \\
\hline $\mathrm{DM}$ & 20.1 & 90.9 \\
\hline $\mathrm{OM}$ & 91.1 & 93.5 \\
\hline $\mathrm{N}$ & 3.2 & 2.9 \\
\hline $\mathrm{NDF}$ & 31.3 & 39.9 \\
\hline Sucrose & 8.64 & 6.78 \\
\hline Total fatty acids (FA) & 2.1 & 0.8 \\
\hline \multicolumn{2}{|l|}{ Individual FA } & - \\
\hline $16: 0$ & 21.4 & 29.9 \\
\hline $18: 0$ & 3.9 & 5.6 \\
\hline cis-9 18:1 & 3.2 & 4.0 \\
\hline cis-11 18:1 & 0.48 & 0.84 \\
\hline trans 18:1 & $\mathrm{ND}^{1}$ & 0.04 \\
\hline cis-9, cis-12 18:2 & 17.1 & 19.0 \\
\hline $18: 3$ & 46.8 & 25.7 \\
\hline
\end{tabular}

${ }^{1} \mathrm{ND}=$ not detected.

ratory under anaerobic conditions at $39^{\circ} \mathrm{C}$. The ruminal fluid was blended to dislodge particle-associated bacteria and strained through 8 layers of cheesecloth. We have previously observed that this procedure allows small particles to be present in the inoculum. The inoculum was divided into 2 equal volumes, and either SB or WB was added at $20 \%$ of total volume. Inocula were gassed with $\mathrm{CO}_{2}$ and blended while $30 \mathrm{~mL}$ was transferred to each of the centrifuge tubes containing the various substrates (Piwonka and Firkins, 1993, 1996).

Each set of 8 treatments was incubated for $0,1,2$, $3,4,6,9$, and $12 \mathrm{~h}$. Times were chosen to identify peak concentrations of CLA and VA in early hours and concomitantly with a lower standard error of means previously reported (Ribeiro and Eastridge, 2004). After incubation, $\mathrm{pH}$ was measured and the tubes were placed in ice to stop fermentation. The tube contents were transferred to aluminum pans, frozen, freezedried, and kept at $-80^{\circ} \mathrm{C}$ until analyses were performed.

\section{Analysis of FA}

The FA of substrates and residues of incubation were methylated with $2 \mathrm{~mL}$ of $0.5 \mathrm{~mol} / \mathrm{L}$ sodium methoxide $\left(10 \mathrm{~min}\right.$ at $\left.50^{\circ} \mathrm{C}\right)$, followed by $3 \mathrm{~mL}$ of $5 \%$ methanolic $\mathrm{HCl}\left(10 \mathrm{~min}\right.$ at $\left.80^{\circ} \mathrm{C}\right)$ as described by Kramer et al. (1997). Methyl esters were separated by gas chromatography using a HP 5890 Series II gas chromatograph (Hewlett Packard Co., Palo Alto, CA). The column was a fused silica capillary (SP-2560, $100 \mathrm{~m} \times 0.25 \mathrm{~mm}$ i.d. $\times 0.2 \mu \mathrm{m}$ film thickness; Supelco, Inc., Bellefonte, PA). Helium was used as carrier gas. Detector and injector temperatures were $260^{\circ} \mathrm{C}$, and the split ratio was 80:1. Oven temperature was $166^{\circ} \mathrm{C}$ for $39 \mathrm{~min}$, increased by $10.0^{\circ} \mathrm{C} / \mathrm{min}$ to $240^{\circ} \mathrm{C}$, held for $10 \mathrm{~min}$, increased by 


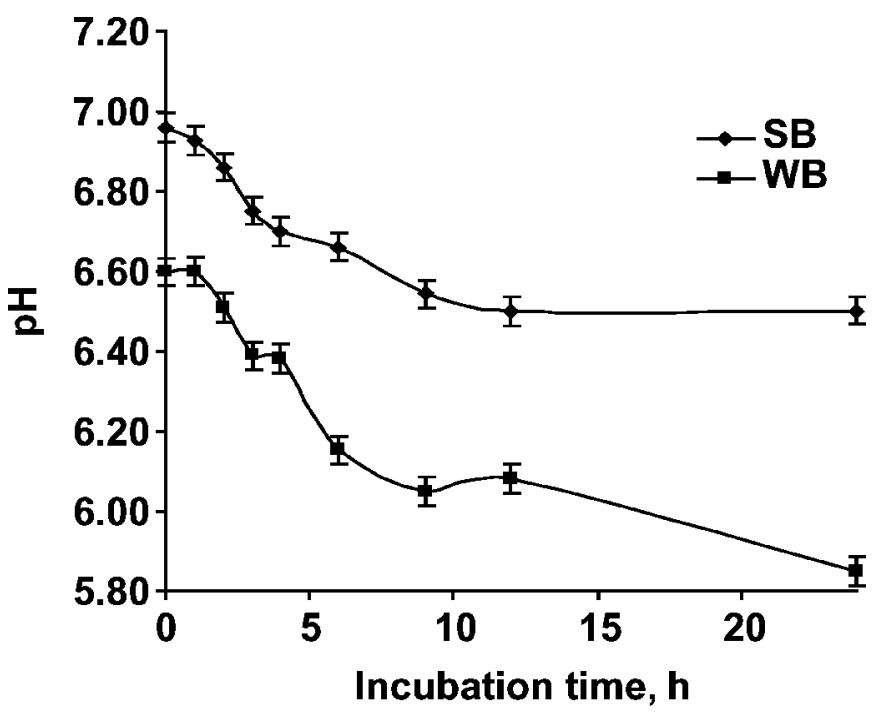

Figure 1. Changes in $\mathrm{pH}$ during in vitro incubation of alfalfa hay or fresh alfalfa incubated with a strong (SB) or weak (WB) buffer.

$3.0^{\circ} \mathrm{C} / \mathrm{min}$ to $245^{\circ} \mathrm{C}$, and held for $10 \mathrm{~min}$. The temperature program used was optimized to separate most of the 18:1 FA in the first isothermal range as described by Molkentin and Precht (1995). Nonadecanoic acid was used as an internal standard. Retention times and response factors were determined with methyl ester standards purchased from Nu-Chek Prep (Elysian, MN; cat. no. GLC-60) and Matreya, Inc. (Pleasant Gap, PA; FIMFAME-7). The 18:1 FA that were not available commercially (trans-6/8, trans- 9 , trans- 12 , trans- 13 , trans- 15 , cis-12, and cis-15) were identified by order of elution as shown by Molkentin and Precht (1995).

\section{Statistical Analyses}

Data were first analyzed as a complete randomized block design using PROC MIXED of SAS (SAS Institute, 2004) according to the following model:

$$
\mathrm{Y}_{\mathrm{ijk}}=\mu+\mathrm{A}_{\mathrm{i}}+\mathrm{c}_{\mathrm{j}}+\mathrm{T}_{\mathrm{k}}+\mathrm{AT}_{\mathrm{ik}}+\varepsilon_{\mathrm{ijk}},
$$

where $Y_{\mathrm{ijk}}=$ dependent variable for treatment $\mathrm{i}$ on block $\mathrm{j}$ and time $\mathrm{k} ; \mu=$ overall mean; $\mathrm{A}_{\mathrm{i}}=$ fixed effect of treatment $\mathrm{i} ; \mathrm{i}=1,2,3,4,5,6,7,8 ; \mathrm{c}_{\mathrm{j}}=$ random effect of block $\mathrm{j} ; \mathrm{j}=1,2 ; \mathrm{T}_{\mathrm{k}}=$ fixed effect of time $\mathrm{k} ; \mathrm{k}=1,2$, $3,4,5,6,7,8 ; \mathrm{AT}_{\mathrm{ik}}=$ interaction of treatment $\mathrm{i}$ with time $\mathrm{k}$; and $\varepsilon_{\mathrm{ijk}}=$ residual error associated with the ijkth observation.

In model [1], there is no functional form imposed on the effect of time and time by treatment interaction.

\section{Single Available Pool, First-Order Kinetic Model}

The first-order kinetic model of Ørskov and McDonald (1979) was fitted with the PROC NLIN procedure of SAS (SAS Institute, 2004) using the least squares subclass means from [1] as observations. The model for estimating $\mathrm{BH}$ rates of 18:3 and 18:2 was as follows:

$$
\mathrm{Y}=\mathrm{C}+\mathrm{Pe}^{-\mathrm{k}(\mathrm{t}-\mathrm{l})}+\varepsilon_{\mathrm{i}},
$$

where $\mathrm{Y}=$ amount $(\mathrm{mg} / \mathrm{tube})$ of $\mathrm{FA}$ at time $\mathrm{t} ; \mathrm{C}=$ pool of unavailable FA; $\mathrm{P}=$ pool of potentially available FA; $\mathrm{k}=$ fractional rate of FA disappearance $\left(\mathrm{h}^{-1}\right) ; \mathrm{t}=$ incubation time (h); $1=$ lag $(\mathrm{h})$; and $\varepsilon_{\mathrm{i}}=$ residual error associated with the ith observation.

Model [2] was fit independently of each of the 8 treatments. The $\mathrm{k}$ and $\mathrm{l}$ parameter estimates for each treatment were compared by orthogonal contrasts to test the effects of buffer, level of sucrose (linear and quadratic), forage source, and interactions between buffer and sucrose, and buffer and forage source. Significance was declared at $P<0.05$ and trends at $P<0.15$.

\section{Multiple Pool, First-Order Kinetic Model}

The time effect can be modeled alternatively by using the more complex kinetic diagram with multiple pools. To do so, the least squares subclass means of model [1] were used as observations to estimate fractional rates of transfer among FA pools using SAAM II software (SAAM, 1997). Observations (least squares subclass means of [1]) were weighted by the reciprocals of their standard errors. The concentrations of the intermediates of $\mathrm{BH}$ from 0 to $12 \mathrm{~h}$ were used also in SAAM II to estimate transfer rates among the pools. The decision to specify direct transfer among specific pools was made using 2 sequential steps: 1 ) if there were published data showing a direct link for the pools, and 2) the addition of a pool or transfer produced a smaller value of the Akaike information criterion (AIC; Cobelli and Foster, 1998):

$$
\mathrm{AIC}=\mathrm{WRSS}+2 \mathrm{P},
$$

where WRSS = weighted residual sum of squares, and $\mathrm{P}=$ number of unknown model parameters.

There is evidence that cis-9, trans-11 18:2 (one of the CLA isomers) is the first product of the $\mathrm{BH}$ of 18:2 (Kepler et al., 1966; Noble et al., 1974); therefore, the pool of 18:2 has a transfer rate to the CLA pool. The Rosenbrock integrator method (SAAM, 1997) was used and the optimization was performed using a variance model based on relative data and a forward derivative. Because there are no data on the transfer rate values 

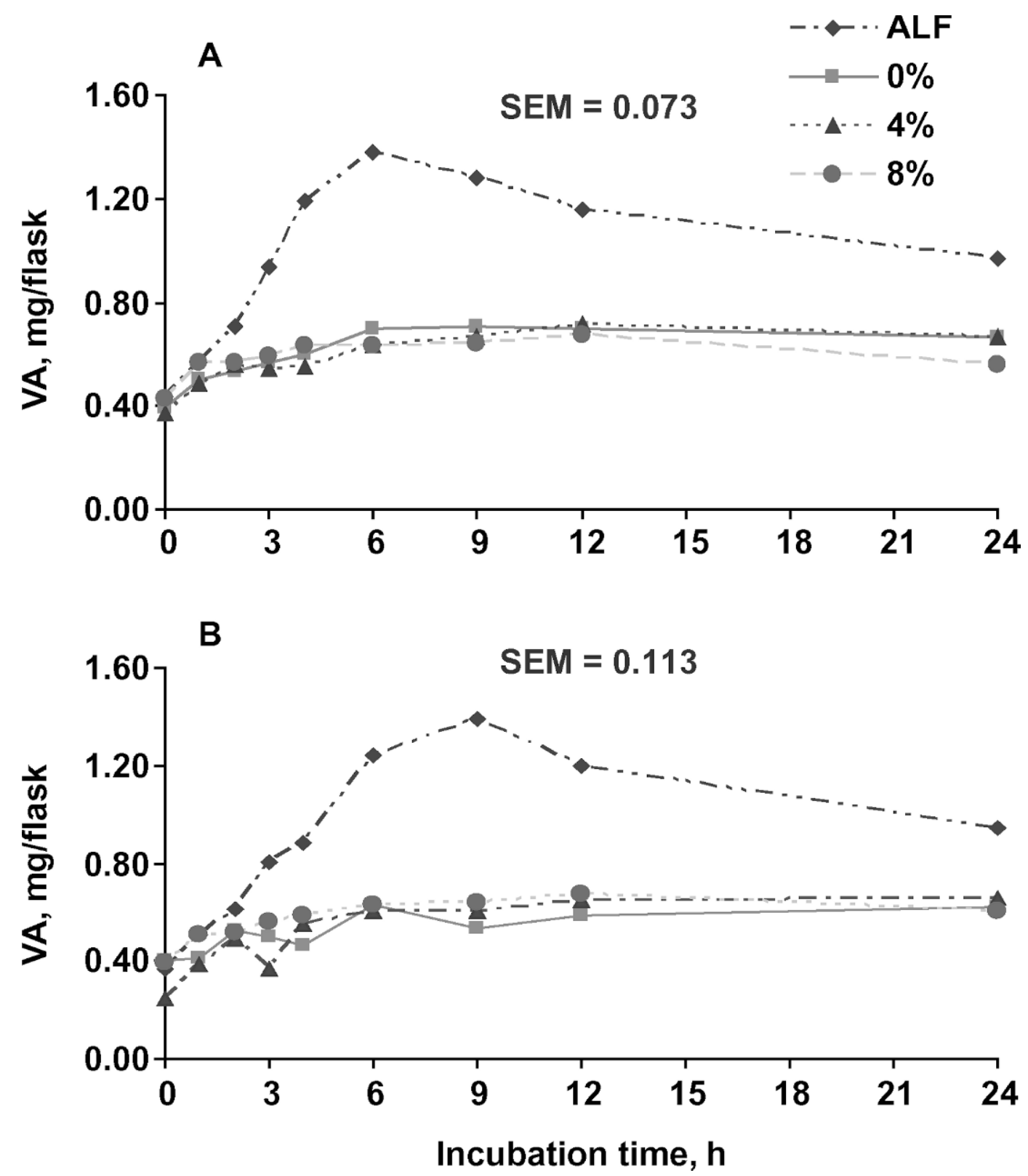

Figure 2. Changes in vaccenic acid (VA; trans-11 18:1) concentration over time during incubation with A) strong, or B) weak buffer. Treatments were fresh alfalfa (ALF) and alfalfa hay with addition of 0,4 , or $8 \%$ sucrose.

for the intermediates of $\mathrm{BH}$, the initial parameter estimates for the $\mathrm{BH}$ rates of $18: 2$ and 18:3 were the same as the ones generated by the equation [1]. Means of $\mathrm{BH}$ rates were compared using the paired difference test (Snedecor and Cochran, 1980).

\section{RESULTS}

The temporal patterns of $\mathrm{pH}$ in the incubation media with the 2 buffers across forages are shown in Figure 1. The mean $\mathrm{pH}$ for the SB was 6.7 and did not fall below 6.4, whereas the mean $\mathrm{pH}$ for the $\mathrm{WB}$ was 6.3 , and it dropped below 6.2 after $5 \mathrm{~h}$ of incubation.

Because we did not separate free and esterified FA in the incubated tubes, the $\mathrm{BH}$ values reported represent a combination of lipolysis and $\mathrm{BH}$. Assuming the lipolysis rate is much faster than $\mathrm{BH}$ rates (Harfoot and Hazlewood, 1997), the $\mathrm{BH}$ values are used to represent the disappearance of 18:2 and 18:3 by hydrogenation after their release from glycerides by lipolysis. Lag times for both 18:2 and 18:3, and the C fraction (unavailable FA) were not different from zero (data not shown). There 
Table 2. Fractional rates (k) of disappearance of $18: 2$ and $18: 3\left(\mathrm{~h}^{-1}\right)$ during in vitro incubation of fresh alfalfa and alfalfa hay in strong (SB) or weak buffer (WB) and with or without supplemental sucrose, determined by a single model

\begin{tabular}{|c|c|c|c|c|c|c|c|c|c|c|}
\hline \multirow[b]{4}{*}{ Item } & \multicolumn{8}{|c|}{ Treatment } & \multirow[b]{4}{*}{$\mathrm{SEM}^{1}$} & \multirow{4}{*}{$\begin{array}{l}\text { Treatment } \\
\text { effects }^{2} \\
(P<0.05)\end{array}$} \\
\hline & & & & & $\mathrm{Hay}+\mathrm{s}$ & rose $(\%$ & & & & \\
\hline & \multicolumn{2}{|c|}{ Fresh alfalfa } & \multicolumn{2}{|c|}{0} & \multicolumn{2}{|c|}{4} & \multicolumn{2}{|c|}{8} & & \\
\hline & WB & SB & WB & SB & WB & SB & WB & SB & & \\
\hline $18: 2$ & 0.202 & 0.361 & 0.174 & 0.404 & 0.143 & 0.403 & 0.156 & 0.250 & 0.058 & B \\
\hline 18:3 & 0.355 & 0.404 & 0.163 & 0.435 & 0.203 & 0.378 & 0.201 & 0.338 & 0.063 & $\mathrm{~B} ; \mathrm{F}$ \\
\hline
\end{tabular}

${ }^{1}$ Average SEM across treatments.

${ }^{2}$ Orthogonal contrasts to test the effects of buffer (B; all WB vs. all SB treatments) and forage (F; all fresh alfalfa vs. all alfalfa hay treatments). There was no effect $(P>0.15)$ of sucrose and there was a trend $(P<$ 0.15 ) for the interaction between forage and buffer.

was a trend $(P=0.11)$ for an interaction between buffer and forage source for the $\mathrm{BH}$ rates of 18:3 (Table 2); the $\mathrm{BH}$ rates of $18: 3$ from fresh alfalfa decreased $12 \%$ compared with a $62 \%$ decrease from alfalfa hay. There was no effect $(P>0.15)$ of sucrose addition on the BH rates of $18: 2$ and 18:3 (Table 2 ).

The representation of the FA pools used to estimate the fractional rates in the multiple pool model during ruminal BH is shown in Figure 3. Because the pool size was low for many of the intermediates during the $\mathrm{BH}$ of FA from hay, the estimation of fractional rates of transfer among pools became unreliable or did not resolve; thus, only the fresh alfalfa data were used. The CLA isomers and the intermediates of 18:3 were not measured; however, we could estimate the temporal changes of the CLA pool and used one FA pool to represent cis-9, trans-11, cis-15 octadecatrienoic acid plus trans-11, cis-15 octadecadienoic acid (18:3-Int pool; Figure 3$)$. The $\mathrm{BH}$ rates for $18: 2$ and 18:3 were lower $(P<$ 0.05 ; Figure 3) for WB than for SB, consistent with the rates estimated using the single pool kinetic model. There was no difference (14.1 and $12.5 ; P>0.15)$ in the $\mathrm{BH}$ rate of VA between the $\mathrm{WB}$ and $\mathrm{SB}$, although the $\mathrm{BH}$ rates of 18:2 and 18:3 were decreased by the WB. Conversely, the WB treatment decreased $(P<0.05)$ the $\mathrm{BH}$ rates of the 18:3-Int to trans-18:1 isomers (trans9, trans-10, trans-12, and trans-13 pool) and the latter to stearic acid.

The flux to and from the VA pools for the fresh alfalfa treatments calculated from SAAM II data are illustrated in Figure 4. The convergence of the input and output curves of the VA pool represents the time of peak VA concentration. The flux from the VA pool was approximately 27\% higher for the SB between 3 to $6 \mathrm{~h}$ of incubation. Because the $\mathrm{BH}$ rate of $18: 3$ was greater for the SB compared with the WB, the flux to the VA pool peaked earlier for the SB.

\section{DISCUSSION}

Fresh alfalfa was used as a positive control to establish standard values for accumulation of VA and other intermediates of $\mathrm{BH}$ in the effluent and to compare differences in $\mathrm{BH}$ that could be attributed to forage conservation. The use of fresh alfalfa with a lower percentage of NDF compared with hay was done intentionally to represent the selection of the immature parts of the plant by grazing cows (Table 1 ).

\section{Effects of pH, Sucrose, and Forage Source on $\mathrm{BH}$}

Because the WB treatment resulted in a lower $\mathrm{pH}$ than the SB, we considered that the main buffer effect was a result of changes in $\mathrm{pH}$. Lower $\mathrm{pH}$ is associated with an impairment of lipolysis (Van Nevel and Demeyer, 1996), BH, and lower fiber digestibility (Qiu et al., 2004). The decrease in $\mathrm{BH}$ with lower $\mathrm{pH}$ may be associated with a decrease in the numbers of cellulolytic bacteria (Wales et al., 2004), because cellulolytic bacteria play a major role in ruminal $\mathrm{BH}$ and these are known to be decreased at lower $\mathrm{pH}$ (Harfoot and Hazlewood, 1997). A pH of 6.2 is considered to be the lower threshold for optimum fiber digestion (Grant and Weidner, 1992), although the period of time that $\mathrm{pH}$ is less than 6.2 may be more significant in inhibiting growth of cellulolytic bacteria (Calsamiglia et al., 2002). Moreover, low pH inhibits lipolysis (Van Nevel and Demeyer, 1996), decreasing $\mathrm{BH}$ by diminishing the availability of free UFA. Therefore, the lower BH rates for 18:2 and 18:3 with the WB treatment were expected. Qiu et al. (2004) observed reduced cellulolytic bacterial numbers with reduced $\mathrm{BH}$ of $18: 2$ and a $50 \%$ decrease in total $\mathrm{BH}$ when the $\mathrm{pH}$ changed from 6.5 to 5.8 in continuous culture. Kolver and de Veth (2002) reported that the ruminal $\mathrm{pH}$ of grazing cows may be as low as 5.8. Because low $\mathrm{pH}$ may increase the proportion of intermedi- 
A

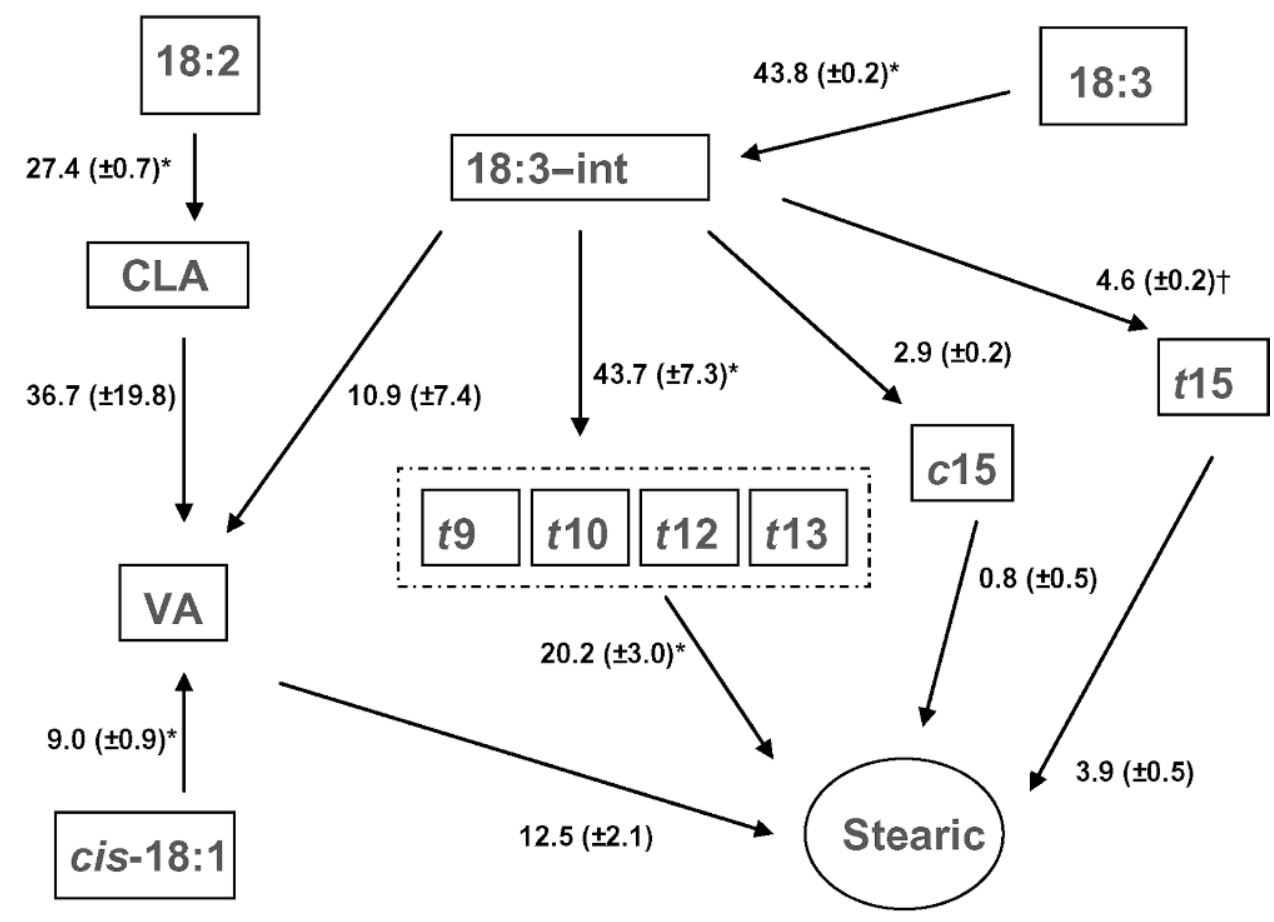

B

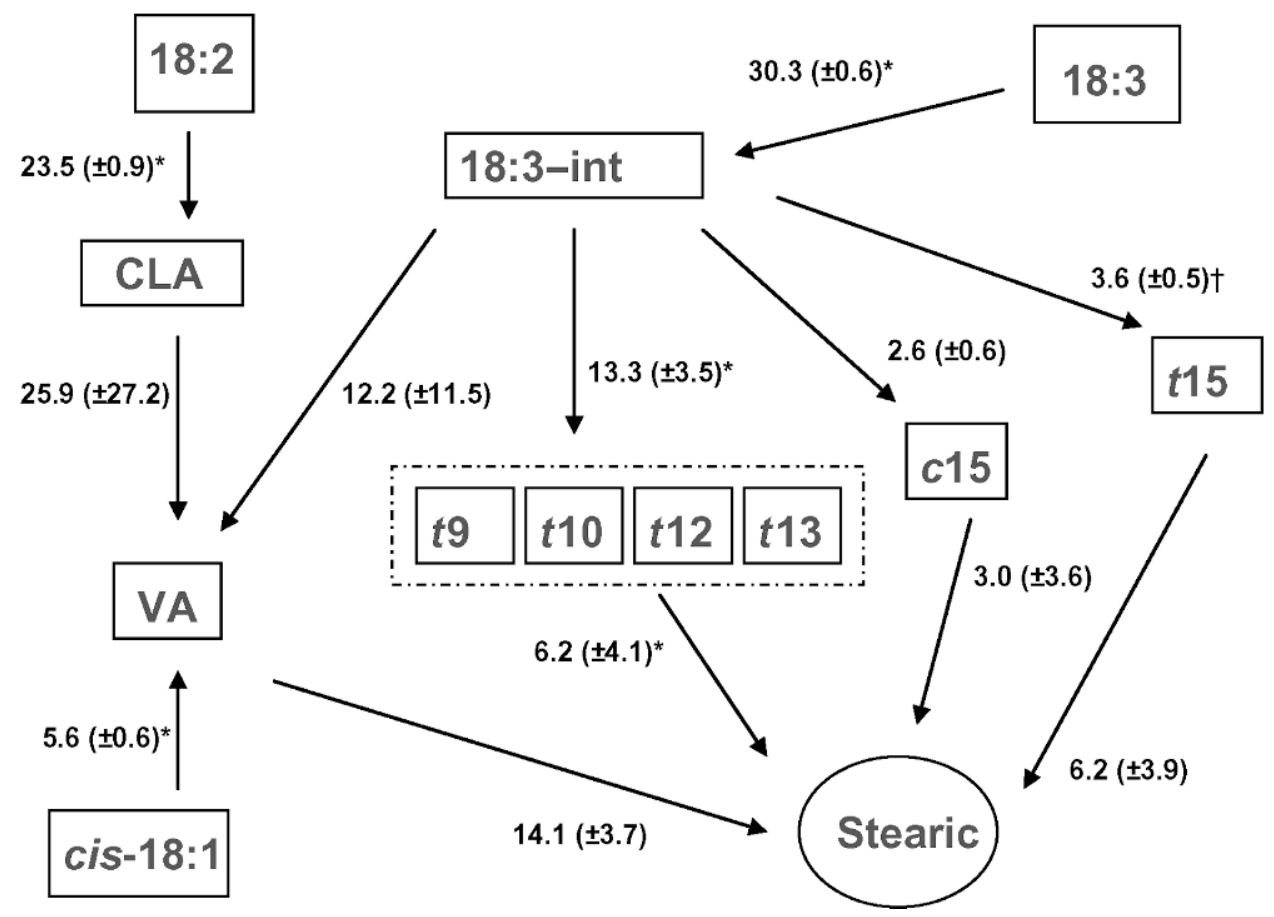

Figure 3. Model of in vitro ruminal biohydrogenation of fatty acids from fresh alfalfa from 0 to $12 \mathrm{~h}$ incubated with A) strong buffer or B) weak buffer. Boxes represent fatty acid pools and arrows represent rates $(\% / \mathrm{h})$ of transfer between pools during biohydrogenation with the standard error in parentheses. Linolenic acid (18:3); linoleic acid (18:2); conjugated linoleic acid isomers (CLA); cis-9, trans-11, cis-15 octadecatrienoic acid plus trans-11, cis-15 octadecadienoic acid (18:3-Int); vaccenic acid (VA); cis-9 octadecenoic acid (cis-18:1); cis-15 octadecenoic acid (cis-15); trans-15 octadecenoic acid (trans-15); trans-9 plus trans-10 plus trans-12 plus trans-13 octadecenoic acids (trans-9, trans10, trans-12, and trans-13); and stearic acid (Stearic). ${ }^{*} P<0.05 ; \dagger P<0.10$. 

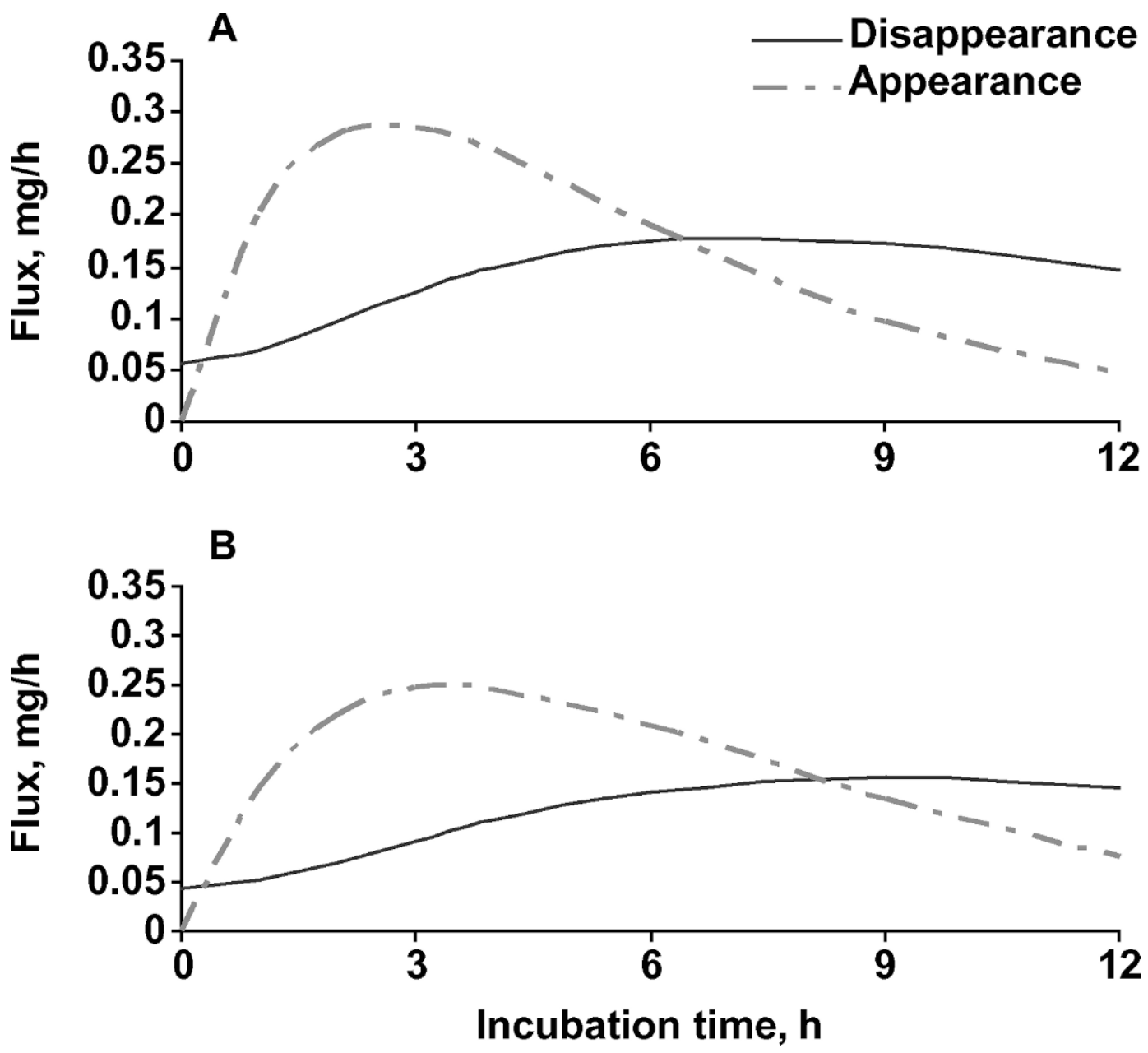

Figure 4. Estimation of the instantaneous flux $(\mathrm{mg} / \mathrm{h})$ to and from the vaccenic acid pool from a multiple pool, first-order kinetic model (SAAM II) during in vitro biohydrogenation of fresh alfalfa with A) strong and B) weak buffer.

ates of $\mathrm{BH}$ (Qiu et al., 2004), the diurnal variation in $\mathrm{pH}$ of grazing ruminants and the high 18:3 concentration of pasture could increase the flow of VA out of the rumen. Others have shown that lower $\mathrm{pH}$ decreases lipolysis (Van Nevel and Demeyer, 1996) and the extent of BH, and increases the proportion of monoenoic FA in the medium (Van Nevel and Demeyer, 1996; Griinari et al., 1998; Troegeler-Meynadier et al., 2003). The BH rates of 18:3 was almost always higher than the rates of $18: 2$ and it was $54 \%$ higher when fresh alfalfa, compared with hay, was incubated with the WB. Thus, fresh alfalfa may be able to maintain the number of some cellulolytic (biohydrogenating) species even at decreased $\mathrm{pH}$.

We did not observe any lag time during $\mathrm{BH}$ of 18:2 and 18:3. Zero lag times have been reported previously with incubation of forages (Boufaied et al., 2003) and oil seeds (Enjalbert et al., 2003). Conversely, TroegelerMeynadier et al. (2003) reported lag times between 1 and $2 \mathrm{~h}$ for soybean oil. Lag time is related to the time needed to release the glyceride from its matrix and is dependent on fat source (Beam et al., 2000) and might be associated with DM digestibility and time for the microbes to adapt to the substrate and incubation conditions. The UFA of fresh plants are located mainly in the chloroplasts as mono- and digalactosyldiglycerides (Butler and Bailey, 1973; Hawke, 1973), and hydrolysis of plant galactolipids has been reported to be 78 to $95 \%$ complete after $4 \mathrm{~h}$ of incubation (Dawson and Hemington, 1974). In fact, rapid hydrolysis of galactoglycerides and triglycerides (Beam et al., 2000) may explain the lack of lag time observed. Additionally, unesterified FA at $0 \mathrm{~h}$ in the incubation tubes would have contributed to the differences in lag time observed among experiments.

Boufaied et al. (2003) reported higher rates of 18:3 $\mathrm{BH}$ for fresh grass compared with hay, supporting our findings. Those authors argued that, because dry hay may have a lower DM digestibility, the FA could have 
been trapped physically within the organic matrix, decreasing rate of release, and therefore, decreasing $\mathrm{BH}$ of $18: 2$ and 18:3. Because the conservation process decreases the absolute and relative amounts of UFA of fresh forages (Chilliard et al., 2000), the percentage of FA in fresh alfalfa was 1.6 times higher than that for alfalfa hay (Table 1). Therefore, one expected outcome in nongrazing cows is reduced concentration and outflow of 18:3 FA from the rumen.

Sucrose had no effect on the rates of $\mathrm{BH}$ of $18: 2$ and 18:3; this observation did not support our hypothesis that sucrose would inhibit $\mathrm{BH}$. We postulated that sucrose incubated with WB would result in greater impairment of $\mathrm{BH}$ than when incubated with $\mathrm{SB}$, but we observed no interaction $(P>0.05)$ between sucrose and buffer source on the $\mathrm{BH}$ rates of 18:2 and 18:3. Conversely, continuous input of sucrose has been shown to impair the $\mathrm{BH}$ of 18:2 and 18:3 from alfalfa hay in continuous culture by a $\mathrm{pH}$-independent process (Ribeiro et al., 2005). Perhaps, the lack of an adaptation period under in vitro conditions as occurs with continuous culture may have caused the different responses in rates of $\mathrm{BH}$ between the 2 methods of incubation.

\section{Intermediates of $\mathrm{BH}$}

Because the proportion of 18:2 in forages is low compared with traditional diets used in nongrazing conditions, the amount of CLA in the incubation tubes was too low to be measured accurately; therefore, CLA concentrations are not reported. The concentration of VA was highest with the fresh alfalfa treatment (Figure 2 ), which was expected because there was $79 \%$ more 18:3 in fresh alfalfa than in alfalfa hay. In addition, the majority of the VA may originate from $\mathrm{BH}$ of 18:3 when forage is the only source of FA (Sudarshan and Hawke, 1979).

Changes in pool size of VA over time reflect relative rates of appearance (synthesis) and disappearance (hydrogenation). During in vitro BH of FA from fresh alfalfa, the VA concentration peaked with the same magnitude at $7 \mathrm{~h}$ for the $\mathrm{SB}$ and $9 \mathrm{~h}$ for the $\mathrm{WB}$, followed by a decrease in its concentration until $12 \mathrm{~h}$ (Figure 2). Similar BH patterns of VA during in vitro incubation have been demonstrated by Noble et al. (1974), who provided $0.32,0.65$, and $0.97 \mathrm{mg} / \mathrm{mL}$ of $18: 2$ and observed VA to peak at 1 and $2 \mathrm{~h}$ of incubation for the 2 lowest concentrations of 18:2, respectively. Qiu et al. (2004) reported no effect of $\mathrm{pH}$ on the proportion of VA in the effluent of continuous culture fermenters. The authors speculated that the lack of effect of $\mathrm{pH}$ on VA flow was a result of a continuous input of 18:2. In our hay treatments, the VA curves did not increase sharply, as observed for the fresh alfalfa treatment, perhaps because the initial amount of UFA available for $\mathrm{BH}$ was much lower with hay. Troegeler-Meynadier et al. (2003) observed a higher rate of appearance of the intermediates of $\mathrm{BH}$ when a higher amount of 18:2 was provided. Additionally, the biohydrogenating microflora seems to be much more active in fresh alfalfa compared with alfalfa hay (Ribeiro et al., 2005).

The trans-13/14 18:1 FA had the second highest concentration of all 18:1 intermediates. Loor et al. (2003) reported that VA was the primary intermediate of $\mathrm{BH}$, followed by trans-13 18:1 FA during continuous culture fermentation of grasses. The procedure to identify FA used in this trial did not allow the separation of trans13 from trans-14 FA. The synthesis of both FA from 18:3 $\mathrm{BH}$ has been reported previously (Ward et al., 1964; White et al., 1970); however, the exact pathway and microorganisms involved are not known.

\section{Kinetics of $\mathrm{BH}$}

Most of the research with in vitro $\mathrm{BH}$ used nonlinear regression and simple first-order kinetics to estimate the $\mathrm{BH}$ rates of $18: 2$ and 18:3, whereas no studies have reported $\mathrm{BH}$ rates of $\mathrm{VA}$ and other intermediates of $\mathrm{BH}$ (Beam et al., 2000; Enjalbert et al., 2003; TroegelerMeynadier et al., 2003). The factors that control the concentration of VA during ruminal $\mathrm{BH}$ are significant because VA plays a central role in $\mathrm{BH}$ (Harfoot and Hazlewood, 1997), and it is the major source of CLA in milk of grazing cows (Kay et al., 2004). Modeling ruminal BH is a useful tool to characterize the influence of diet, microbial ecology, and ruminal factors on the $\mathrm{BH}$ process, and to postulate potential $\mathrm{BH}$ pathways. However, there are limited data in the literature in which the $\mathrm{BH}$ rates of the intermediates of $\mathrm{BH}$ have been estimated. Therefore, we developed a multicompartmental kinetic model to estimate the fractional rates of VA appearance and disappearance to characterize the influence of dietary and ruminal factors on pool size and flux of VA during in vitro $\mathrm{BH}$.

First-order kinetics has been used previously to estimate $\mathrm{BH}$ rates of UFA from different sources (Beam et al., 2000; Boufaied et al., 2003; Troegeler-Meynadier et al., 2003). A second-order kinetic model would be a much more difficult model to integrate and develop. We used first-order kinetics in our model because we expected that the UFA concentration would be below saturates because of the low percentage of FA in the alfalfa samples (2\%) such that pseudo first-order kinetics would apply. On pools that we evaluated, the natural logarithm concentration vs. time resulted in a linear plot (first order), and the inverse of concentration vs. time was not linear (i.e., not second order). Nonetheless, future research should evaluate if a multicompartmen- 

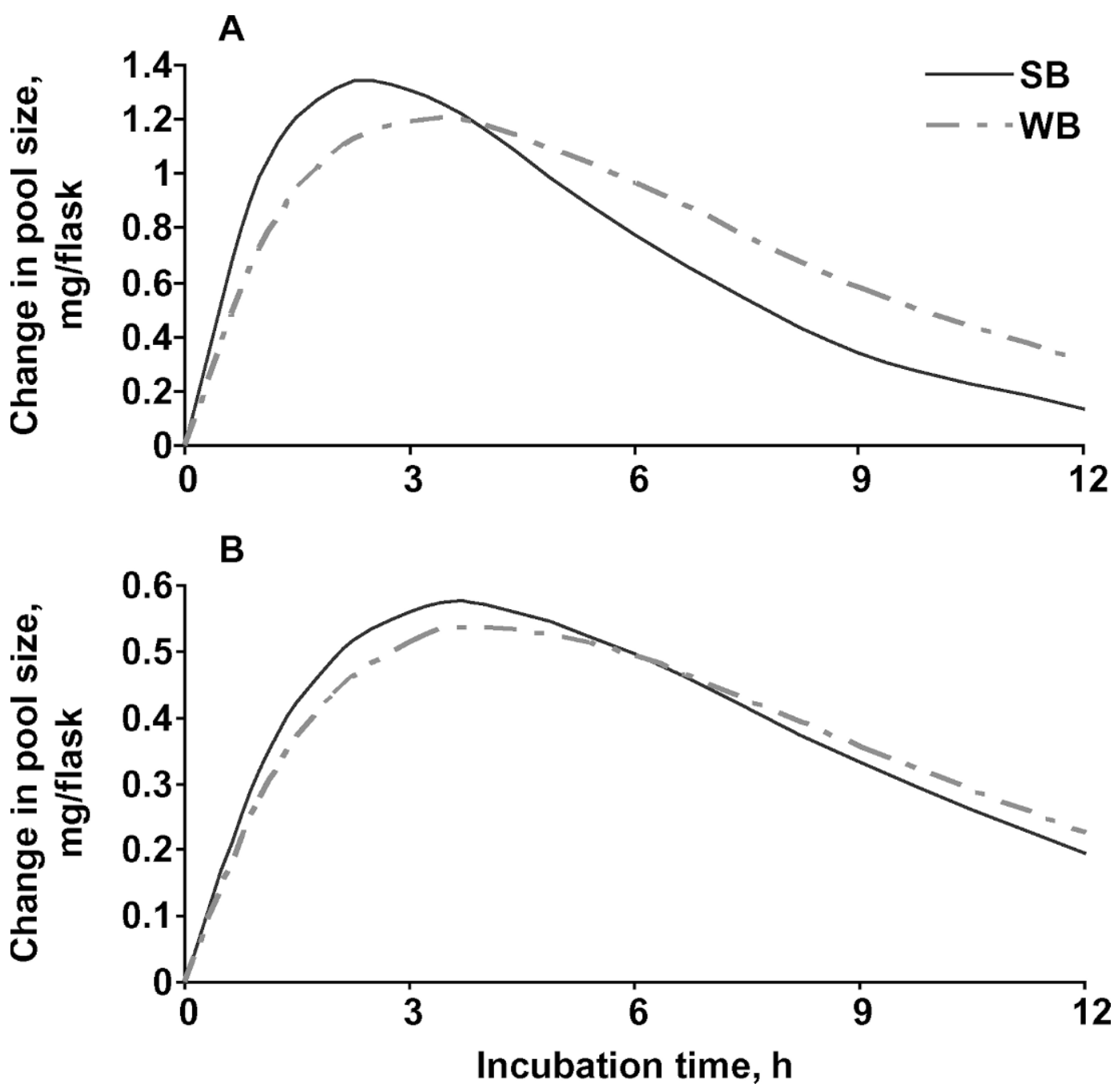

Figure 5. Representation of the temporal change of the pool size (mg) of A) cis-9, trans-11, cis-15 octadecatrienoic acid plus trans-11, cis-15 octadecadienoic acid (18:3-Int pool), and B) conjugated linoleic acid estimated from a multiple pool, first-order kinetic model (SAAM II) during in vitro biohydrogenation of fresh alfalfa with strong (SB) or weak (WB) buffer.

tal, first-order kinetic model would be ideal when other substrates are utilized.

The WB treatment slowed the $\mathrm{BH}$ of the 18:3-Int pool (Figure 3), and after $4 \mathrm{~h}$, the 18:3-Int pool size was greater for the WB treatment, consistent with the lower $\mathrm{BH}$ rate of $18: 3$ and may be consistent also with the later peak of VA observed for the WB (Table 2 and Figure 2). The temporal changes in the CLA and 18:3Int pool sizes are illustrated in Figure 5. The 18:3-Int pool increased more sharply when fresh alfalfa was incubated with the SB than with WB. Also, the 18:3Int pool decreased faster than the CLA pool, regardless of the buffer used, because the $\mathrm{BH}$ rates of 18:3 was faster than for 18:2. Additionally, the CLA and 18:3Int pools had higher $\mathrm{BH}$ rates compared with the 18:1 isomers, which is consistent with previous reports that those FA do not accumulate during ruminal $\mathrm{BH}$ as much as 18:1 isomers (Noble et al., 1974; Body, 1976); they are also associated with the same group of biohydrogenating bacteria (Harfoot and Hazlewood, 1997), reflecting the faster $\mathrm{BH}$ rates. The variation of the CLA concentration over time followed the same pattern as reported previously (Noble et al., 1974; Kellens et al., 1986) and was similar to the VA curve, but peaked earlier, consistent with precursor and product kinetics.

We are not aware of any other research reporting $\mathrm{BH}$ of VA without using isotopes. To measure the accuracy of the multicompartmental model approach in estimating pool size of VA, we compared the concentration of VA observed (the least squares subclass means from [1]) and estimated by the model (Figure 6). The coefficient of variation for the VA curves was 3.8 and $5.2 \%$ for the 

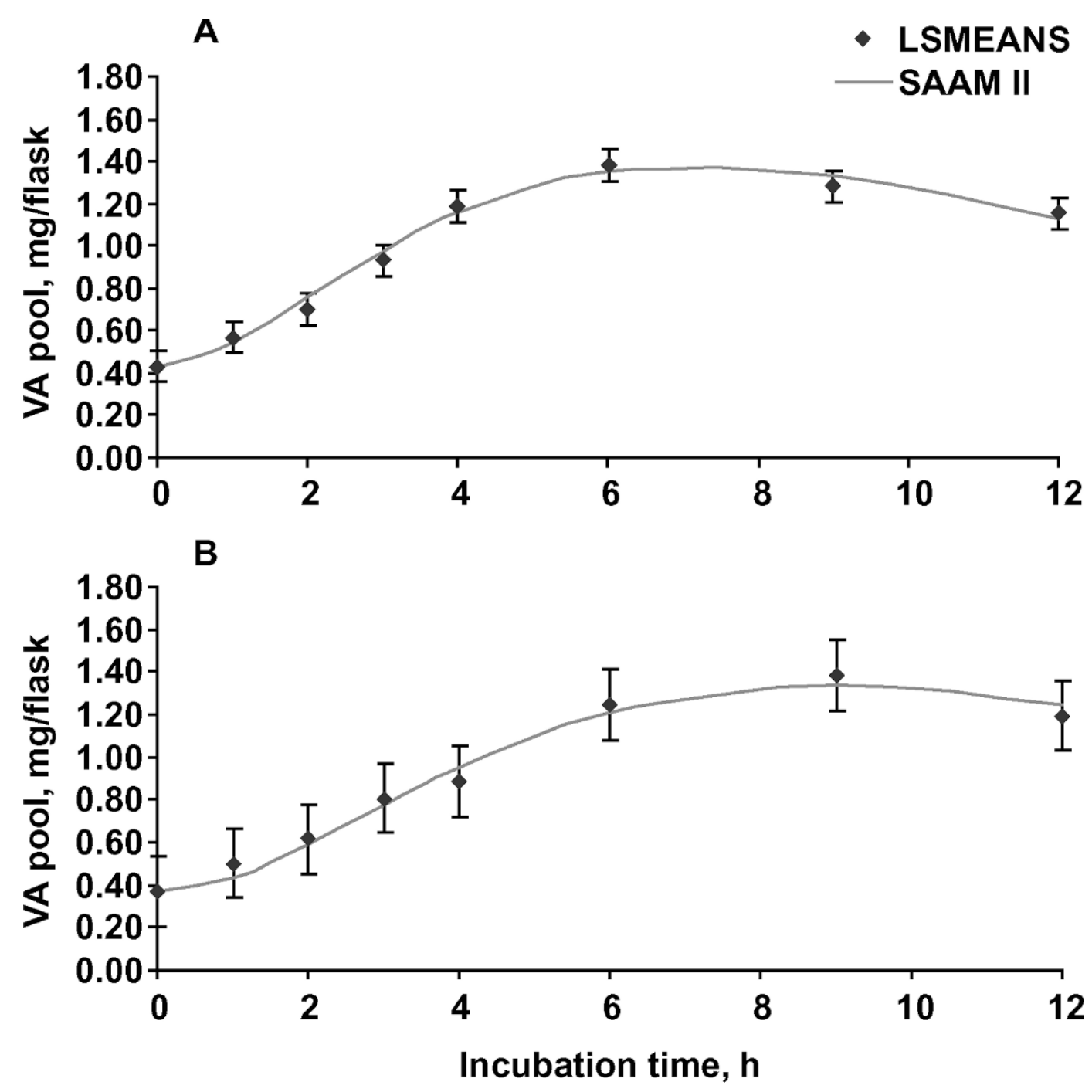

Figure 6. Changes in the in vitro vaccenic acid (VA) pool: least squares means from randomized complete block design analysis (observed) and fit from SAAM II (estimated) from incubations of fresh alfalfa with A) strong or B) weak buffers. The root mean square errors for curves $\mathrm{A}$ and $\mathrm{B}$ were 0.036 and 0.045 , respectively.

SB and WB, respectively. Appearance (synthesis) of VA was greater than disappearance until approximately 6 and $9 \mathrm{~h}$ for SB and WB, respectively. These represent the times that VA concentration peaked for each buffer (Figure 2). Because a multiple pool model (SAAM, 1997) estimates fluxes, as well as mass of the VA pools, it generates more information from the data than a single pool model (SAS Institute, 2004).

The $\mathrm{BH}$ rates of 18:2 and 18:3 estimated by the compartmental model are within the range of previously published data (Enjalbert et al., 2003; Troegeler-Meynadier et al., 2003). Additionally, Moate et al. (2004) estimated $\mathrm{BH}$ rates of $\mathrm{FA}$ using in vivo studies; the authors reported that the $\mathrm{BH}$ rate of trans-18:1 isomers was $22.8 \% / \mathrm{h}$, very similar to the $\mathrm{BH}$ rate of the trans-
18:1 isomers pool for the SB $(20.2 \% / \mathrm{h})$. However, future research is needed to determine how methodology (in vivo vs. in vitro) and dietary treatments affect disappearance rates of the intermediates of $\mathrm{BH}$.

The flux of appearance of VA was higher during the first $6 \mathrm{~h}$ of incubation (Figure 4). This flux was estimated by summing the fluxes from the 18:3-Int, CLA, and cis-18:1 pools to the VA pool as illustrated in our model (Figure 3). Although the transfer rates are similar between appearance and disappearance of VA, the higher flux of appearance resulted from the higher mass of the 18:3-Int pool during the early time points. The high standard error for the BH rate of CLA and 18:3Int to VA (Figure 3 ) is a reflection of their high correlation $(r=-0.70$; data not shown). Additionally, because 
we were unable to set a direct link between 18:2-Int and CLA pools (due to overparameterization of the model), the standard error may have also been inflated.

The last step of $\mathrm{BH}$ is assumed to be most sensitive to $\mathrm{pH}$ because trans-18:1 FA accumulate when highgrain diets are fed (Griinari et al., 1998). However, the WB decreased the rates of $\mathrm{BH}$ of 18:2 and 18:3 without affecting the mean concentration and $\mathrm{BH}$ rate of VA. A shift to alternate pathways of $\mathrm{BH}$ that do not include VA could explain the lack of response of VA concentration to lower $\mathrm{pH}$. For instance, WB decreased $(P<0.05$; Figure 3 ) the $\mathrm{BH}$ of other 18:1 FA pools (trans-9, trans10 , trans -12 , and trans -13 ) to 18:0 and the hydrogenation of the 18:3-Int pool to the other 18:1 FA pool. Consequently, the lower rate of disappearance of $18: 2$ and 18:3 caused a more pronounced decrease in the flux of the UFA to other trans FA while maintaining almost similar fluxes to VA.

\section{Limitations of the Modeling Approach}

Although using a multicompartmental model to estimate $\mathrm{BH}$ is superior to a single-compartmental approach to model the dynamics of FA metabolism in the rumen, there are constraints and assumptions that limit interpretation. One constraint is that all rate and pool estimates are correlated and any error in estimating one rate will affect others. For instance, the 18:0 pool size may be underestimated; because 18:0 is the final product of $\mathrm{BH}$, any error in estimating the precedent pools will be reflected in the 18:0 pool. Moreover, any intermediate of $\mathrm{BH}$ and microbial FA synthesis not taken into account that contributes to the synthesis of 18:0 will increase the error of estimating the pool size of 18:0.

Although some intermediates of $\mathrm{BH}$ of oleic acid have been reported (Ward et al., 1964; Mosley et al., 2002), the pathways and microbial species for $\mathrm{BH}$ of oleic acid have not been defined. Therefore, defining direct links between pools may be compromised. For example, we assumed that cis- and trans-15 18:1 were hydrogenated to 18:0; however, Harfoot and Hazlewood (1997) considered these to be true end products of 18:3 BH. However, there is still the possibility that cis- and trans-15 18:1 are in fact hydrogenated directly to $18: 0$, because we can not rule out this step based on the research done by Body (1976) and White et al. (1970). Including hydrogenation of cis- and trans-15 18:1 to 18:0 in the model improved the fit of the model (AIC value) because the concentration of both decreased after $9 \mathrm{~h}$ of incubation. Alternatively, these may have been isomerized to other 18:1 FA (Mosley et al., 2002; Proell et al., 2002) and then hydrogenated to 18:0; however, the model under this option did not solve.
Compartmental analysis of $\mathrm{BH}$ of FA in fresh alfalfa showed that the mean concentration and $\mathrm{BH}$ rate of VA were not affected by buffer, whereas the last step of $\mathrm{BH}$ of the WB treatment was slower for other trans18:1 FA, and the mean concentration of trans-10 18:1 was higher. The concentration of trans- and cis-15 18:1 FA declined, most likely due to hydrogenation to 18:0, an observation not previously reported. Our data demonstrated the need to define even more the intermediates of $\mathrm{BH}$ (such as individual CLA isomers), as well the fractional rates of transfer among pools as dietary management is changed and microbial populations shift. Such knowledge would greatly improve understanding of the complex reactions that occur in the rumen and that affect the profile of FA in ruminant products.

\section{CONCLUSIONS}

Culturing rumen contents at lower $\mathrm{pH}$ decreased $\mathrm{BH}$ rates of $18: 2$ and 18:3, independent of the forage source with a greater decrease in the $\mathrm{BH}$ rates of 18:3 for alfalfa hay than for fresh alfalfa. Sucrose did not affect $\mathrm{BH}$ rates of $18: 2$ and 18:3. There was no interaction between sucrose and incubation $\mathrm{pH}$ on the rates of $\mathrm{BH}$ of $18: 2$ and $18: 3$.

We quantified changes in the rates of $\mathrm{BH}$ of trans18:1 FA and VA, which is the key regulatory step determining the amount of VA leaving the rumen and affecting concentration of milk CLA. The multicompartmental model analysis allowed factors affecting the $\mathrm{BH}$ rates of $18: 2$ and 18:3 to be defined, as well as the synthesis and hydrogenation rates of other $\mathrm{BH}$ intermediates. We demonstrated the magnitude of $\mathrm{BH}$ pathways, other than through VA, that may explain variations in VA concentration as incubation conditions change. Because we could estimate fluxes, as well as mass of the VA pools, more information was generated from the data when using multiple pools compared with a single pool, first-order kinetic model. Future research should focus on increasing the number of individual FA pools that can be estimated by the model and quantify changes on $\mathrm{BH}$ rates with dietary and ruminal factors.

\section{REFERENCES}

Beam, T. M., T. C. Jenkins, P. J. Moate, R. A. Kohn, and D. L. Palmquist. 2000. Effects of amount and source of fat on the rates of lipolysis and biohydrogenation of fatty acids in ruminal contents. J. Dairy Sci. 83:2564-2573.

Body, D. R. 1976. The occurrence of cis-octadec-15-enoic acid as a major biohydrogenation product from methyl linolenate in bovine rumen liquor. Biochem. J. 157:741-744.

Boufaied, H., P. Y. Chouinard, G. F. Tremblay, H. V. Petit, R. Michaud, and G. Belanger. 2003. Fatty acids in forages. II. In vitro ruminal biohydrogenation of linolenic and linoleic acids from timothy. Can. J. Anim. Sci. 83:513-522. 
Butler, G. W., and R. W. Bailey. 1973. Chemistry and biochemistry of herbage. Academic Press, London, UK.

Calsamiglia, S., A. Ferret, and M. Devant. 2002. Effects of $\mathrm{pH}$ and $\mathrm{pH}$ fluctuations on microbial fermentation and nutrient flow from a dual-flow continuous culture system. J. Dairy Sci. 85:574-579.

Chilliard, Y., A. Ferlay, R. M. Mansbridge, and M. Doreau. 2000. Ruminant milk fat plasticity: Nutritional control of saturated, polyunsaturated, trans and conjugated fatty acids. Ann. Zootech. 49:181-205.

Cobelli, C., and D. M. Foster. 1998. Compartmental models: Theory and practice using SAAM II software system. Page 423 in Mathematical Modeling in Experimental Nutrition. A. J. Clifford and H. Muller, ed. Plenum Press, New York, NY.

Dawson, R. M., and N. Hemington. 1974. Digestion of grass lipids and pigments in the sheep rumen. Br. J. Nutr. 32:327-340.

Enjalbert, F., P. Eynard, M. C. Nicot, A. Troegeler-Meynadier, C. Bayourthe, and R. Moncoulon. 2003. In vitro versus in situ ruminal biohydrogenation of unsaturated fatty acids from a raw or extruded mixture of ground canola seed/canola meal. J. Dairy Sci. 86:351-359.

French, P., C. Stanton, F. Lawless, E. G. O’Riordan, F. J. Monahan, P. J. Caffrey, and A. P. Moloney. 2000. Fatty acid composition, including conjugated linoleic acid, of intramuscular fat from steers offered grazed grass, grass silage, or concentrate-based diets. J. Anim. Sci. 78:2849-2855.

Grant, R. J., and S. J. Weidner. 1992. Digestion kinetics of fiber: Influence of in vitro buffer $\mathrm{pH}$ varied within observed physiological range. J. Dairy Sci. 75:1060-1068.

Griinari, J. M., D. A. Dwyer, M. A. McGuire, D. E. Bauman, D. L. Palmquist, and K. V. V. Nurmela. 1998. Trans-octadecenoic acids and milk fat depression in lactating dairy cows. J. Dairy Sci. 81:1251-1261.

Harfoot, C. G., and G. P. Hazlewood. 1997. Lipid metabolism in the rumen. Pages 382-426 in The Rumen Microbial Ecosystem. 2nd ed. P. N. Hobson, ed. Elsevier, London, UK.

Hawke, J. C. 1973. Chemistry and biochemistry of herbage. Pages 213-263 in Lipids. G. W. Butler and R. W. Bailey, ed. Academic Press, London, UK.

Jahreis, G., J. Fritsche, and H. Steinhart. 1997. Conjugated linoleic acid in milk fat: High variation depending on production system. Nutr. Res. 17:1479-1484.

Kay, J. K., T. R. Mackle, M. J. Auldist, N. A. Thomson, and D. E. Bauman. 2004. Endogenous synthesis of cis-9, trans-11 conjugated linoleic acid in dairy cows fed fresh pasture. J. Dairy Sci. 87:369-378.

Kellens, M. J., H. L. Goderis, and P. P. Tobback. 1986. Biohydrogenation of unsaturated fatty acids by a mixed culture of rumen microorganisms. Biotechnol. Bioeng. 37:1268-1276.

Kelly, M. L., E. S. Kolver, D. E. Bauman, M. E. Van Amburgh, and L. D. Muller. 1998. Effect of intake of pasture on concentrations of conjugated linoleic acid in milk of lactating cows. J. Dairy Sci. 81:1630-1636.

Kepler, C. R., K. P. Hirons, J. J. McNeill, and S. B. Tove. 1966. Intermediates and products of the biohydrogenation of linoleic acid by Butyrivibrio fibrisolvens. J. Biol. Chem. 241:1350-1354.

Kolver, E. S., and M. J. de Veth. 2002. Prediction of ruminal $\mathrm{pH}$ from pasture-based diets. J. Dairy Sci. 85:1255-1266.

Kramer, J. K., V. Fellner, M. E. Dugan, F. D. Sauer, M. M. Mossoba, and M. P. Yurawecz. 1997. Evaluating acid and base catalysts in the methylation of milk and rumen fatty acids with special emphasis on conjugated dienes and total trans fatty acids. Lipids $32: 1219-1228$.

Loor, J. J., W. H. Hoover, T. K. Miller-Webster, J. H. Herbein, and C. E. Polan. 2003. Biohydrogenation of unsaturated fatty acids in continuous culture fermenters during digestion of orchardgrass or red clover with three levels of ground corn supplementation. J. Anim. Sci. 81:1611-1627.

Moate, P. J., W. Chalupa, T. C. Jenkins, and R. C. Boston. 2004. A model to describe ruminal metabolism and intestinal absorption of long chain fatty acids. Anim. Feed Sci. Technol. 112:79-105.

Molkentin, J., and D. Precht. 1995. Optimized analysis of transoctadecenoic acids in edible fats. Chromatographia 41:267-272.

Mosley, E. E., G. L. Powell, M. B. Riley, and T. C. Jenkins. 2002. Microbial biohydrogenation of oleic acid to trans isomers in vitro. J. Lipid Res. 43:290-296.

Noble, R. C., J. H. Moore, and C. G. Harfoot. 1974. Observations on the pattern on biohydrogenation of esterified and unesterified linoleic acid in the rumen. Br. J. Nutr. 31:99-108.

Ørskov, E. R., and J. McDonald. 1979. The estimation of protein degradability in the rumen from incubation measurements weighed according to rate of passage. J. Agric. Sci. (Camb.) 92:499-503.

Piwonka, E. J., and J. L. Firkins. 1993. Effect of glucose on fiber digestion and particle-associated carboxymethylcellulase activity in vitro. J. Dairy Sci. 76:129-139.

Piwonka, E. J., and J. L. Firkins. 1996. Effect of glucose fermentation on fiber digestion by ruminal microorganisms in vitro. J. Dairy Sci. 79:2196-2206.

Proell, J. M., E. E. Mosley, G. L. Powell, and T. C. Jenkins. 2002. Isomerization of stable isotopically labeled elaidic acid to cis and trans monoenes by ruminal microbes. J. Lipid Res. 43:2072-2076.

Qiu, X., M. L. Eastridge, K. E. Griswold, and J. L. Firkins. 2004. Effects of substrate, passage rate, and $\mathrm{pH}$ in continuous culture on flows of conjugated linoleic acid and trans C18:1. J. Dairy Sci. 87:3473-3479.

Ribeiro, C. V. D. M., and M. L. Eastridge. 2004. Relationship of rate of appearance of vaccenic acid and $\mathrm{pH}$ during in vitro biohydrogenation of linolenic acid in alfalfa hay. J. Dairy Sci. 83(Suppl. 1):37. (Abstr.)

Ribeiro, C. V. D. M., S. K. R. Karnati, and M. L. Eastridge. 2005. Biohydrogenation of fatty acids and digestibility of fresh alfalfa or alfalfa hay plus sucrose in continuous culture. J. Dairy Sci. 88:4007-4017.

SAAM. 1997. SAAM II User Guide. SAAM Inst., Seattle, WA.

SAS Institute. 2004. SAS/STAT User's Guide. Version 8 ed. SAS Institute Inc, Cary, NC.

Snedecor, G. W., and W. G. Cochran. 1980. Statistical Methods. 7th ed. Iowa State University Press, Ames.

Sudarshan, S., and J. C. Hawke. 1979. The in vitro lipolysis and biohydrogenation of monogalactosyldiglyceride by whole rumen contents and its fractions. J. Sci. Food Agric. 30:603-612.

Troegeler-Meynadier, A., M. C. Nicot, C. Bayourthe, R. Moncoulon, and F. Enjalbert. 2003. Effects of $\mathrm{pH}$ and concentrations of linoleic and linolenic acids on extent and intermediates of ruminal biohydrogenation in vitro. J. Dairy Sci. 86:4054-4063.

Van Nevel, C. J., and D. I. Demeyer. 1996. Influence of $\mathrm{pH}$ on lipolysis and biohydrogenation of soybean oil by rumen contents in vitro. Reprod. Nutr. Dev. 36:53-63.

Van Soest, P. J. 1994. Nutritional ecology of the ruminant. Cornell University Press, Ithaca, NY.

Wales, W. J., E. S. Kolver, P. L. Thorne, and A. R. Egan. 2004. Diurnal variation in ruminal $\mathrm{pH}$ on the digestibility of highly digestible perennial ryegrass during continuous culture fermentation. J. Dairy Sci. 87:1864-1871.

Ward, P. F., T. W. Scott, and R. M. Dawson. 1964. The hydrogenation of unsaturated fatty acids in the ovine digestive tract. Biochem. J. 92:60-68.

White, R. W., P. Kemp, and M. C. Dawson. 1970. Isolation of a rumen bacterium that hydrogenates oleic acid as well as linolenic acid and linoleic acid. Biochem. J. 116:767-768.

Woolford, M. K. 1984. The Silage Fermentation. Vol. 14. Microbiology Series ed. Marcel Dekker, Inc., New York, NY. 